Employee of: Galapagos, Jörg H.W. Distler Shareholder of: 4D Science, Consultant of: Actelion, Active Biotech, Anamar, ARXX, Bayer Pharma, Boehringer Ingelheim, Celgene, Galapagos, GlaxoSmithKline, Inventiva, JB Therapeutics, Medac, Pfizer, RuiYi and UCB, Grant/research support from: Anamar, Active Biotech, Array Biopharma, ARXX, aTyr, Bristol Myers Squibb, Bayer Pharma, Boehringer Ingelheim, Celgene, Galapagos, GlaxoSmithKline, Inventiva, Novartis, Sanofi-Aventis, RedX and UCB

DOI: 10.1136/annrheumdis-2021-eular.1839

\section{OP0243 AN APPROACH COMBINING TRANSCRIPTOMIC AND TOPOGRAPHIC ANALYSIS REVEALS A POTENTIAL ROLE OF PROTEASOME AND AUTOPHAGY DEREGULATION IN THE PATHOPHYSIOLOGY OF DERMATOMYOSITIS}

L. Debrut ${ }^{1}$, G. Laverny ${ }^{2}$, P. Mertz ${ }^{3}$, M. Giannini ${ }^{4}$, M. Pizzimenti5, A. Charlot5, C. Keime $^{6}$, B. Lannes ${ }^{7}$, D. Metzger ${ }^{2}$, B. Geny ${ }^{8}$, J. Sibilia ${ }^{4}$, A. Meyer ${ }^{4} .{ }^{1}$ Centre de Recherche en Biomédecine de Strasbourg, UR3072, mitochondrie, stress oxydant et protection musculaire, Strasbourg, France; ${ }^{2}$ IGBMC, IGBMC, IIIkirch, France: ${ }^{3} \mathrm{CHU}$ Strasbourg, Immunology, Strasbourg, France: ${ }^{4} \mathrm{CHU}$ Strasbourg, Rhumatologie, Strasbourg, France; ${ }^{1}$ Centre de Recherche en Biomédecine de Strasbourg, UR3072, mitochondrie, stress oxydant et protection musculaire, Strasbourg, France; ${ }^{6}$ GGBMC, Genomeast, Illkirch, France; ${ }^{7} \mathrm{CHU}$ Strasbourg, Anatomopathologie, Strasbourg, France: ${ }^{8} \mathrm{CHU}$ Strasbourg, Explorations fonctionnelles, Strasbourg, France

Background: Dermatomyositis (DM) is a rare autoimmune muscle disease characterized by an atrophy and a type-l interferon signature in perifascicular fibers. We recently showed that muscle mitochondrial dysfunction is also a characteristic of this disease that participates to both decrease exercise capacity and maintenance of inflammation (1). Pathophysiological mechanisms underlying these characteristics are unknown.

Objectives: The objective of this study is to reveal the mechanisms underlying the modifications of perifascicular fibers during DM, taking advantage of a method combining transcriptomic and topographical information.

Methods: Fourteen patients with recent ( $<6$ months) untreated myositis (DM: $n=7$, other myositis: $n=7$ ) who underwent a biopsy of the deltoid muscle for diagnostic purposes were included. Seven other patients with suspected but not confirmed neuromuscular pathology (normal creatine kinase level, electromyogram and deltoid biopsy) were also included (no myopathy: $n=7$ ). Under the control of optical microscopy, perifascicular fibers (about 400 fibers) and endofascicular fibers (about 400 fibers) were microdissected by laser. The transcriptome of endofascicular fibers and perifascicular fibers in all three groups of patients were then obtained by massive sequencing of total messenger RNA. The DAVID database (2) (http://david.abcc.ncifcrf.gov) were used to determine the deregulated molecular pathways in the perifascicular fibers during DM

Results: 482 transcripts were differently expressed in perifascicular fibers of patients with DM compared to perifascicular fibers of the 2 other groups (348 overexpressed and 134 underexpressed). The most overexpressed transcripts were involved in the type I interferon response while the most underexpressed transcripts were involved in mitochondria and in proteasome functioning. The study of the transcripts differentially expressed in perifasicular versus endofascicular fibers revealed that there is a physiological perifascicular signature: in patients without myopathy, 83 genes were overexpressed and 54 were underexpressed in perifascicular fibers compared to endofascicular fibers. This physiological perifascicular signature was abolished in patient with myositis (DM and other myositis). In the group of patients with DM (but not other myositis), a specific perifascicular signature (18 genes overexpressed and 10 genes underexpressed in perifascicular fibers compared to endofascicular fibers) was identified. The most deregulated transcripts in DM perifascicular fibers were involved in autophagy/mitophagy, mitochondria and proteasome pathways.

Conclusion: In the physiological state, perifascicular fibers are characterized by a different transcriptomic profile from endofascicular fibers. During DM, this physiological perifascicular signature is abolished and replaced by a transcriptomic signature that reveal a potential role of proteasome and autophagy deregulation in the pathophysiology of DM.

\section{REFERENCES:}

[1] Meyer et al. IFN- $\beta$-induced reactive oxygen species and mitochondrial damage contribute to muscle impairment and inflammation maintenance in dermatomyositis Acta Neuropathol. 2017;134:655-666.

[2] Huang, D. W. et al. The DAVID Gene Functional Classification Tool: a novel biological module-centric algorithm to functionally analyze large gene lists. Genome Biol 8, R183 (2007).

Disclosure of Interests: None declared

DOI: 10.1136/annrheumdis-2021-eular.2586

\section{OP0244 28 NEW AUTOANTIBODIES AGAINST GPCR, GROWTH FACTORS AND GROWTH FACTOR RECEPTORS ARE ASSOCIATED WITH DISEASE MANIFESTATIONS IN SYSTEMIC SCLEROSIS}

K. Sterner ${ }^{1}$, C. J. K. Fouodo ${ }^{2}$, I. König ${ }^{2}$, A. Künstner ${ }^{3}$, H. Busch ${ }^{3}$, H. Heidecke ${ }^{4}$, A. Schumann ${ }^{5}$, A. Müller ${ }^{5}$, G. Riemekasten ${ }^{1}$, S. Schinke ${ }^{5} .{ }^{1}$ University of Lübeck, Department of Rheumatology and Clinical Immunology, Lübeck, Germany; ${ }^{2}$ University of Lübeck, Institute of Medical Biometry and Statistics, Lübeck, Germany; ${ }^{3}$ University of Lübeck, Lübeck Institute of Experimental Dermatology, Lübeck, Germany; ${ }^{4} \mathrm{CellTrend} \mathrm{GmbH,} \mathrm{-,} \mathrm{Luckenwalde,} \mathrm{Germany;}{ }^{5} \mathrm{UKSH}$ Campus Lübeck, Department of Rheumatology and Clinical Immunology, Lübeck, Germany

Background: The morbidity and mortality of systemic sclerosis (SSc) are largely determined by vascular and fibrotic pathologies. Levels of autoantibodies (ab) against G protein-coupled receptors (GPCR), growth factors (GF) and growth factor receptors (GFR) are altered in SSc compared to healthy controls $(\mathrm{HC})$ ${ }^{1}$. Thus, higher angiotensin II receptor type 1 - (AT1R) and endothelin receptor type A - (ETAR) ab levels are associated with severe disease and SSc-related mortality ${ }^{2}$. CXC motiv chemokine receptor 3 - (CXCR3) and 4 - (CXCR4) ab have predictive value for deterioration of pulmonary fibrosis $(\mathrm{PF})^{3}$.

Objectives: We used statistical methods to identify associations between disease manifestations and 28 new ab directed against GPCR, GF and GFR in SSc. Methods: Ab against the following targets were measured in sera from SSc patients $(n=177)$ and $\mathrm{HC}(n=88)$ : Adrenoceptors alpha-1 (ADRA1), alpha-2 (ADRA2), beta-1 (ADRB1), beta-2 (ADRB2); muscarinoceptors 1-5 (M1R M5R); AT1R, ETAR, endothelin B receptor (ETBR); CXCR3, CXCR4; complement receptors $3 \mathrm{a}(\mathrm{C} 3 \mathrm{aR})$ and $5 \mathrm{a}(\mathrm{C5aR})$; protease-activated receptors 1 (PAR1) and 2 (PAR2); vascular endothelial growth factor A (VEGFA) and its receptors 1 (VEGFR1) and 2 (VEGFR2), epithelial growth factor (EGF)/ - receptor (EGFR); hepatocyte growth factor (HGF)/ - receptor (HGFR), platelet-derived growth factor-AA (PDGFAA), placental growth factor (PIGF).

The organ involvement (PF, cardiac involvement, $\mathrm{PAH}$, gastrointestinal tract) and quantitative markers (modified Rodnan skin score, SSc activity score, pulmonary function, cardiac enzymes and echocardiography, routine laboratory, autoimmune diagnostics) as well as demographic data were recorded retrospectively at the time of sample collection. Statistical analysis was performed using the Mann-Whitney $U$ test (MWU), Pearson correlations, ROC analysis, and age-adjusted logistic regression models.

Results: In SSc 20 of 28 measured ab levels are significantly altered compared to HC. According to the Pearson correlation matrix, the ab-levels are highly correlated and build a network that differs between $\mathrm{HC}$ and SSc. Furthermore, altered network signatures are formed in the differentiated analysis of several disease manifestations of SSc such as SSc-subtype or PF. Based on ROC analysis, FGF-ab, ADRB1-ab and PIGF-ab are well suited to predict SSc (Figure 1).

In addition, limited cutaneous SSc (ISSc) patients displayed lower levels of most ab than diffuse cutaneous SSc patients, whereas cardiac and pulmonary involvement are associated with higher ab levels. In the logistic regression ISSc is associated with lower levels of ab against M1R, M2R, C5aR, ETAR, AT1R, PAR1 EGFR. Higher levels for ab against M1R, M2R, ETBR, C5aR are associated with $\mathrm{PF}$, higher levels of ab against complement receptors, adrenoreceptors and EGF with NT-proBNP elevation.

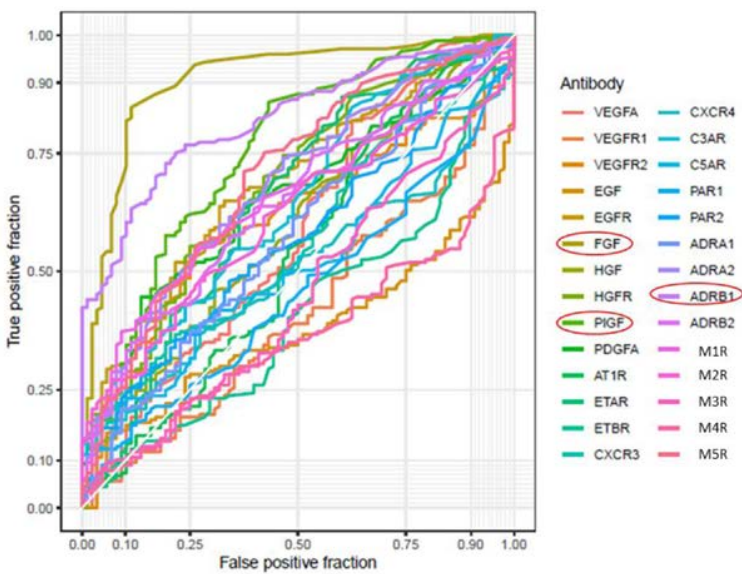

Figure 1: Receiver operating characteristic analyses for 28 new autoantibodies in systemic sclerosis $(n=177)$ compared with healthy controls $(n=88)$. Ab against FGF, ADRB1 and PIGF may predict SSC. 